Pacific Journal of Mathematics

GENERIC SOUSLIN SETS

Vol. 97, No. 1

January 1981 


\title{
GENERIC SOUSLIN SETS
}

\author{
ARNold W. MILLER
}

By iterated forcing we create generic Souslin sets, which we use to answer questions of Ulam, Hansell, and Mauldin. For $X$ a topological space a set $Y \subseteq X$ is analytic in $X$ (also called Souslin in $X$ or $\Sigma_{1}^{1}$ in $X$ ) iff there are Borel sets $B_{s}$ for $s \in \omega^{<\omega}$ such that:

$$
Y=\bigcup_{f \in \omega^{\omega}} \bigcap_{n<\omega} B_{f \nmid n} .
$$

For $X=2^{\omega}$ (the Cantor space) a set $Y \subseteq X$ is analytic iff it is the projection of a Borel subset of $2^{\omega} \times 2^{\omega}$. Given $R \subseteq$ $P(X)$ (the power set of $X$ ) let $B(R)$ be the smallest family of subsets of $X$ including $R$ and closed under countable union and complementation (i.e., the $\sigma$-algebra generated by $R$ ). If $X$ is a topological space and $R$ the family of open sets then $B(R)$ is the family of Borel subsets of $X$. The following question was raised by Ulam.

(1) Does there exist $R \subseteq P\left(2^{\omega}\right)$ such that $R$ is countable and every analytic set in $2^{\omega}$ is an element of $B(R)$ ?

Rothberger showed that assuming $C H$ there is such a $R$. We will show that it is consistent with ZFC that there is no such $R$.

(2) Does there exist a separable metric space $X$ in which every subset is analytic but not every subset is Borel?

This was raised by $\mathrm{R}$. W. Hansell. Clearly $\mathrm{CH}$ implies no such $X$ exists. We show that it is consistent with ZFC that such a $X$ exists.

Let $R=\left\{A \times B: A, B \subseteq 2^{\omega}\right\}$, the abstract rectangles in the plane. Let $S(R)$ be the family of subsets of $2^{\omega} \times 2^{\omega}$ obtained by applying the Souslin operation to sets in $B(R)$. The next question was asked by $\mathrm{D}$. Mauldin.

(3) Does $S(R)=P\left(2^{\omega} \times 2^{\omega}\right)$ imply $B(R)=P\left(2^{\omega} \times 2^{\omega}\right)$ ?

We show that the answer to this question is no.

Preliminaries. Recall the following definitions:

(1) $\omega=\{0,1,2, \cdots\}$ and $\forall n<\omega, n=\{m \mid m<n\}$;

(2) $\omega^{n}=\{s \mid s: n \rightarrow \omega\}$;

(3) for $s \in \omega^{m}$ and $n<\omega, \widehat{s} n$ is that $t \in \omega^{m+1}$ such that $t \uparrow m=s$ and $t(m)=n$;

(4) $\phi$ denotes the empty sequence;

(5) $\omega^{<\omega}=\bigcup\left\{\omega^{n}: n<\omega\right\}$;

(6) $T \subseteq \omega^{<\omega}$ is a tree iff $\forall s, t \in \omega^{<\omega}(s \subseteq t \in T \rightarrow s \in T)$;

(7) $T$ is a well founded tree iff $\forall f \in \omega^{\omega} \exists n<\omega f \uparrow n \notin T$;

(8) for $s \in T$ a well founded tree $|s|_{T}$ is defined inductively by: 


$$
|s|_{T}=\sup \left\{|\hat{s} n|_{T}+1: \exists n \hat{s} n \in T\right\} ;
$$

(9) for $\alpha<\omega_{1}, T$ is a normal $\alpha$-tree iff

(a) $T$ is a well founded tree such that $|\phi|_{T}=\alpha$;

(b) if $s \in T$ and $|s|_{T}>0$, then $\forall n s^{\wedge} n \in T$;

(c) if $s \in T$ and $|s|_{T}=\beta+1$, then $\forall n\left|\hat{s^{\wedge} n}\right|_{T}=\beta$;

(d) if $s \in T$ and $|s|_{T}=\lambda$ where $\lambda$ is a limit ordinal, then $\forall \beta<\lambda$, $\left\{n:\left|s^{\wedge} n\right|_{T}<\beta\right\}$ is finite (see [9]);

(10) for $T \cong \omega^{<\omega}$ a tree define:

$\boldsymbol{P}(T)=\left\{p \mid \exists F \in[T]^{<\omega}, p: F \rightarrow 2, \forall n<\omega, \forall s \in \omega^{<\omega}\right.$, if $s, s^{\wedge} n \in F$, then $p(s)=1$ implies $\left.p\left(s^{\wedge} n\right)=0\right\}, \boldsymbol{P}(T)$ is ordered by inclusion.

(11) A notion of rank on a partial order $\boldsymbol{P}$ is a function whose domain is a subset of $\boldsymbol{P}$ and whose range is the ordinals. For $\alpha$ an ordinal and $p \in \boldsymbol{P}$, we let $|p|=\alpha$ mean that $p$ is in the domain of this function and its value is $\alpha$. The following property must be satisfied. For every $p \in \boldsymbol{P}$ and $\beta \geqq 1$, there exists $\hat{p} \in \boldsymbol{P}$ compatible with $p$ such that $|\hat{p}| \leqq \beta$ and for every $q \in \boldsymbol{P}$ if $|q|<\beta$ and $\hat{p}$ and $q$ are compatible, then $p$ and $q$ are compatible.

(12) Given a notion of rank on $\boldsymbol{P}$ if $\tau$ is a term such that $\mathbb{H}^{\prime \prime} \tau \in 2^{\omega \prime \prime}$, then we say that $|\tau|=0$ iff for any $p \in \boldsymbol{P}$ and $n<\omega$ there exists $q \in \boldsymbol{P}$ compatible with $p$ such that $|q|=0$ and $s \in 2^{n}$ such that $q \Vdash " s s \subseteq \tau^{\prime \prime}$.

(13) For $T$ a normal $\alpha$-tree and $p \in \boldsymbol{P}(T)$ define $|p|$ to be the maximum $|s|_{T}$ for $s \in \operatorname{dom}(p)$.

(14) $T^{*}=\left\{s \in T:|s|_{T}=0\right\}$.

The following lemma is key. It implies that $|p|$ is a rank on $P(T)$.

LEMma 1. $\forall \beta \geqq 1 \forall p \in \boldsymbol{P}(T) \exists \hat{p} \in \boldsymbol{P}(T)$ such that

(a) $p$ and $\hat{p}$ are compatible;

(b) $p \uparrow T^{*}=\hat{p} \uparrow T^{*}$;

(c) $|\hat{p}| \leqq \beta$;

(d) $\forall q \in \boldsymbol{P}(T)$ if $|q|<\beta$, then $\hat{p}$ and $q$ are compatible implies $p$ and $q$ are compatible.

Proof. This is essentially Lemma 2 of [10]. We reprove it here for completeness. Let $F=\left\{s^{\wedge} n: s \in \operatorname{dom}(p), p(s)=1,|s|_{T}=\lambda\right.$ a limit ordinal $>\beta$, and $\left.|\hat{s} n|_{T}<\beta\right\}$. By normality of $T, F$ is finite, and $\forall t \in F,|t|_{T} \geqq 2$. Thus we can find $r \geqq p \forall t \in F \exists m t^{\wedge} m \in \operatorname{dom}(r)$ and $r\left(t^{\wedge} m\right)=1$. Let $D=\left\{s \in \operatorname{dom}(r):|s|_{T} \leqq \beta\right\}$ and $\hat{p}=r \uparrow D . \quad p$ and $\hat{p}$ are compatible since $r$ extends them both. $p \uparrow T^{*}=\hat{p} \uparrow T^{*}$ since $\forall t \in F \forall m\left|t^{\wedge} m\right|_{T} \geqq 1$.

Now we check (d). Suppose $|q|<\beta$ and $p$ and $q$ are not compatible. Then there are $s \in \operatorname{dom}(p)$ and $t \in \operatorname{dom}(q)$ which demonstrate 
that $p \cup q$ is not a condition.

Case 1. $s=t$ and $p(s) \neq q(t)$. Since $|q|<\beta$ it follows $|t|_{T}<\beta$ and so $s \in \operatorname{dom}(\hat{p})$.

Case 2. $s=t^{\wedge} m$ for some $m$ and $p(s)=q(t)=1$. But then $|s|_{T}<|t|_{T}<\beta$ and so again $s \in \operatorname{dom}(\hat{p})$.

Case 3. $t=\hat{s} m$ for some $m$ and $p(s)=q(t)=1$. Since $|t|_{T}<\beta$ either $|s|_{T} \leqq \beta$ and so $s \in \operatorname{dom}(\hat{p})$ or $|s|_{T}=\lambda$ a limit ordinal $>\beta$ in which case $t \in F$ so there exists $n<\omega$ such that $r\left(t^{\wedge} n\right)=1$ and so $t^{\wedge} n \in \operatorname{dom}(\hat{p})$ and so $\hat{p}$ and $q$ are incompatible. In all three cases $\hat{p}$ and $q$ are incompatible.

The next lemma asserts the fact that statements of small rank should be forced by conditions of small rank. $M$ is the ground model of ZFC and $P$ is any partial order with a notion of rank.

LEMMA 2. Let $B(r)$ be any $\boldsymbol{\Sigma}_{\beta}^{0}$ predicate with parameter in $M$, $1 \leqq \beta, \Vdash_{P}^{\prime \prime} \tau \in 2^{\prime \prime},|\tau|=0$, and $p \in \boldsymbol{P}$ such that $p \mathbb{R}^{\prime \prime} B(\tau)^{\prime \prime}$. Then $\exists \hat{p} \in \boldsymbol{P},|\hat{p}|<\beta, p$ and $\hat{p}$ are compatible and $\hat{p} \mathbb{}^{\prime \prime} B(\tau)^{\prime \prime}$.

Proof. The proof is by induction $\beta$.

Case 1. $\beta=1$. Then $p \Vdash^{\prime \prime} \exists n R(\tau \uparrow n, x \uparrow n)^{\prime \prime}$ where $R$ is primitive recursive and $x \in M \cap 2^{\omega}$. Find $q$ extending $p$ and $s \in 2^{n}$ for some $n$ such that $q \Vdash^{\prime \prime} \tau \uparrow n=\breve{s}^{\prime \prime}$ and $R(s, x \uparrow n)$ holds. By the definition of $|\tau|=0, \exists \hat{p}$ compatible with $q$ (and hence with $p$ ) such that $|\hat{p}|=0$ and $\hat{p} \Vdash " \tau \uparrow n=\breve{s}^{\prime \prime}$. Thus $\hat{p} \Vdash " \exists n R(\tau \uparrow n, x \uparrow n)^{\prime \prime}$.

Case 2. $\quad \beta$ a limit ordinal. Then $p \Vdash^{\prime \prime} \exists n B_{n}(\tau)$ " where each $B_{n}(r)$ is a $\boldsymbol{\Sigma}_{\beta_{n}}^{0}$ predicate for some $\beta_{n}<\beta$. Let $p_{0}$ extend $p$ such that $\exists n_{0}<\omega p_{0} \mathbb{H}^{\prime \prime} B_{n_{0}}(\tau)^{\prime \prime}$. By induction $\exists \hat{p}$ compatible with $p_{0}$ (and hence with $p$ ) such that $|\hat{p}|<\beta_{n_{0}}<\beta$ and $\hat{p} \Vdash " B_{n_{0}}(\tau)^{\prime \prime}$ (and hence $\hat{p} \Vdash$ " $\left.\exists n B_{n}(\tau)^{\prime \prime}\right)$.

Case 3. $\beta=\gamma+1$ and $\gamma>0$. As in Case 2 we may as well assume $p \Vdash^{\prime \prime} B(\tau)^{\prime \prime}$ where $B(r)$ is a $\boldsymbol{\Pi}_{r}^{0}$ predicate. By Lemma 1, $\exists \hat{p} \in \boldsymbol{P}, \hat{p}$ and $p$ compatible, $|\hat{p}| \leqq \gamma$, and $\forall q \in \boldsymbol{P}$ if $|q|<\gamma$ and $q$ and $\hat{p}$ are compatible, then $q$ and $p$ are compatible. Then $\hat{p} \Vdash " B(\tau)^{\prime \prime}$. Otherwise $\exists r$ extending $\hat{p}, r \Vdash^{\prime \prime}-B(\tau)^{\prime \prime}$. Since $\neg B(r)$ is a $\boldsymbol{\Sigma}_{r}^{0}$ predicate, by induction $\exists \hat{r} \in \boldsymbol{P},|\hat{r}|<\gamma, \hat{r}$ and $r$ compatible, and $\hat{r} \Vdash^{\prime \prime}-B(\tau)^{\prime \prime}$. But $\hat{r}$ and $p$ are incompatible (since $p \Vdash^{\prime \prime} B(\tau)^{\prime \prime}$ ) and so by choice of 
$\hat{p}, \hat{r}$ and $\hat{p}$ are incompatible a contradiction.

Next we describe almost disjoint forcing (similar to the way it is done in [2]). Given $X=\left\{x_{\alpha}: \alpha<\omega_{1}\right\} \subseteq 2^{\omega}$ distinct and $\left\langle Y_{\alpha}: \alpha<\omega_{1}\right\rangle=$ $Y$ where each $Y_{\alpha} \subseteq \omega^{<\omega}$, we want to force a sequence of $G_{\delta}$ sets $\left\langle G_{s}: s \in \omega^{<\omega}\right\rangle$ such that $\forall s \forall \alpha\left(x_{\alpha} \in G_{s} \leftrightarrow s \in Y_{\alpha}\right)$. Let $B$ be the family of all clopen subsets of $2^{\prime \prime}$. Define $\boldsymbol{P}(X, Y)$ as follows:

it is the set of all $r$ such that

(a) $r$ is a finite subset of $\omega^{<\omega} \times \omega \times(B \cup X)$;

(b) if $\langle s, n, B\rangle,\left\langle s, n, x_{\alpha}\right\rangle \in r$ then $x_{\alpha} \notin B$;

(c) if $\left\langle s, n, x_{\alpha}\right\rangle \in r$ then $s \notin Y_{\alpha}$.

As usual $r$ extends $p,(r \geqq p)$ iff $r \supseteqq p$. It is well known that $P(X, Y)$ satisfies the c.c.c. and also for any $G$ which is $\boldsymbol{P}(X, Y)$-generic if we define $G_{s}=\bigcap_{n} \cup\{B:\{\langle s, n, B\rangle\} \in G\}$ then $\forall s \forall \alpha\left(x_{\alpha} \in G_{s} \leftrightarrow s \in Y_{\alpha}\right)$.

1. Forcing a Souslin set. We now describe how to force Souslin sets. Let $M$ be our ground model of ZFC. Working in $M$ let $F^{*}$ be some standard fixed bijection between $\omega^{<\omega}$ and $\omega$, and define $F: 2^{\omega} \rightarrow 2^{(\omega<\omega)}$ by $F(x)(s)=x\left(F^{*}(s)\right)$. Let $X=\left\{x_{\alpha}: \alpha<\omega_{1}\right\}$ be a fixed subset of $2^{\omega}$ such that for all $\alpha<\omega_{1}, F\left(x_{\alpha}\right)$ is the characteristic function of a normal $\alpha$-tree $T_{\alpha}$. Let

$$
\boldsymbol{P}_{0}=\sum_{\alpha<\omega_{1}} \boldsymbol{P}\left(T_{\alpha}\right)
$$

note that $\boldsymbol{P}_{0}$ has c.c.c. since it is equivalent to adding $\omega_{1}$ Cohen reals. Note that any $G$ which is $\boldsymbol{P}\left(T_{\alpha}\right)$-generic over $M$ determines (and is determined by) a map $G_{\alpha}: T_{\alpha} \rightarrow 2$. $G_{\alpha} \uparrow T_{\alpha}^{*}$ in fact determines $G_{\alpha}$ by the rule $G_{\alpha}(s)=1$ iff $\forall n G_{\alpha}(\hat{s} n)=0$. Given $G^{0} \boldsymbol{P}_{0}$-generic over the ground model $M$, let $G^{0}=\left\langle G_{\alpha}: \alpha<\omega_{1}\right\rangle$ and let $y_{\alpha}=\left\{s \in T_{\alpha}^{*}: G_{\alpha}(s)=0\right\}$. Let $\boldsymbol{P}_{1}=\boldsymbol{P}(X, Y)$ where $Y=\left\langle y_{\alpha}: \alpha<\omega_{1}\right\rangle$. (So $\boldsymbol{P}_{1} \in M\left[G^{0}\right]$.) Let $\boldsymbol{P}=$ $\boldsymbol{P}_{0}^{*} \boldsymbol{P}_{1}$.

Working in $M[G]$ for $G P$-generic over $M$ (so $G=\left(\left\langle G_{\alpha}: \alpha<\omega_{1}\right\rangle\right.$, $\left.\left\langle G^{s}: s \in \omega^{<\omega}\right\rangle\right)$ ) let:

$$
A=\left\{x_{\alpha} \in X: G_{\alpha}(\phi)=1\right\} .
$$

To see that $A$ is analytic in $X$ we will define $\hat{A}$ a $\Sigma_{1}^{1}$ set such that $\hat{A} \cap X=A$. Define $x \in \hat{A}$ iff $\exists T \subseteq \omega^{<\omega}, \exists p: \omega^{<\omega} \rightarrow 2, \exists T^{*} \subseteq \omega^{<\omega}$ such that

(a) $F(x)$ is the characteristic function of $T$;

(b) $T$ is a tree;

(c) $T^{*}=\{s \in T: \exists n \hat{s \wedge} n \notin T\}=\{s \in T: \forall n \widehat{s} n \notin T\}$;

(d) $\forall s \in T^{*} p(s)=1$ iff $x \in G_{s}$;

(e) $\forall s \in T-T^{*} p(s)=1$ iff $\forall n p\left(s^{\wedge} n\right)=0$;

(f) $p(\phi)=1$. 
(a) thru (f) are easily seen to be a Borel predicate of $x, T, T^{*}$, and $p$, and hence $\hat{A}$ is $\boldsymbol{\Sigma}_{1}^{1}$.

In order to show $A$ is a new Souslin set we first want to extend our notion of rank to $\boldsymbol{P}$. Let $\boldsymbol{Q}=\{\boldsymbol{r} \mid \boldsymbol{r}$ satisfies (a) and (b) in the definition of $\boldsymbol{P}(X, Y)\}$ (thus $\boldsymbol{Q} \in M)$. Then

$$
\left\{(p, q): p \in \boldsymbol{P}_{0}, q \in \boldsymbol{Q} \text {, and } p \Vdash^{\prime \prime} \breve{q} \in \boldsymbol{P}(X, Y)^{\prime \prime}\right\}
$$

ordered by $(\hat{p}, \hat{q}) \geqq(p, q)$ iff $\hat{p} \geqq p$ and $\hat{q} \geqq q$, is clearly dense in $\boldsymbol{P}$, so for simplicity assume it is $\boldsymbol{P}$. Let us unravel $p \Vdash^{\prime \prime}$ " $q \in \boldsymbol{P}(X, Y)^{\prime \prime}$. This means that whenever $\left\langle s, n, x_{\alpha}\right\rangle \in q^{\prime}$ then $p \Vdash " s \notin Y_{\alpha}^{\prime \prime}$. But $p \Vdash$

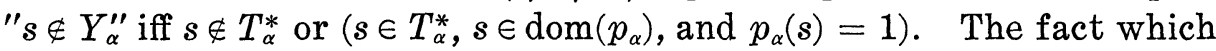
we note is that if $p, p^{\prime} \in \boldsymbol{P}_{0}$ and $\forall \alpha<\omega_{1} p_{\alpha} \uparrow T_{\alpha}^{*}=p_{\alpha}^{\prime} \uparrow T_{\alpha}^{*}$, then $\forall r \in$ $\boldsymbol{Q}\langle p, \boldsymbol{r}\rangle \in \boldsymbol{P}$ iff $\left\langle p^{\prime}, \boldsymbol{r}\right\rangle \in \boldsymbol{P}$.

For any $\alpha<\omega_{1}$, we define the following rank function on $\boldsymbol{P}$ :

$$
|(p, q)|_{\alpha}=\max \left\{|s|_{T_{\gamma}}: \gamma>\alpha \text { and } s \in \operatorname{dom}\left(p_{\gamma}\right)\right\} .
$$

Note that the rank depends only on the part of the condition in $\boldsymbol{P}_{0}$. To see that it is a rank function, let $(p, q)$ be any condition and $\beta \geqq 1$. For each $\gamma>\alpha$ by Lemma $1 \exists \hat{p}_{\gamma} \in \boldsymbol{P}\left(T_{\gamma}\right)$ such that $\hat{p}_{\gamma} \uparrow T_{\gamma}^{*}=$ $p \uparrow T_{r}^{*}, \hat{p}_{\gamma}$ and $p_{\gamma}$ are compatible, $\left|\hat{p}_{\gamma}\right| \leqq \beta$, and $\forall q \in \boldsymbol{P}\left(T_{\gamma}\right)$ if $|q|<\beta$ and $\hat{p}_{r}$ and $q$ are compatible, then $p_{r}$ and $q$ are compatible. Let $\hat{p} \in \boldsymbol{P}_{0}$ be defined by:

$$
\hat{p}_{r}=\left\{\begin{array}{lll}
p_{\gamma} & \text { if } & \gamma \leqq \alpha \\
\hat{p}_{\gamma} & \text { if } & \gamma>\alpha .
\end{array}\right.
$$

By what we have already remarked

$$
\begin{gathered}
(\hat{p}, q) \in \boldsymbol{P},|(\hat{p}, q)|_{\alpha} \leqq \beta,(p, q) \text { and }(\hat{p}, q) \text { are compatible, } \\
\forall\left(p^{\prime}, q^{\prime}\right) \in \boldsymbol{P} \text { if }\left|\left(p^{\prime}, q^{\prime}\right)\right|_{\alpha}<\beta \text { and }
\end{gathered}
$$

$\left(p^{\prime}, q^{\prime}\right)$ is compatible with $(\hat{p}, q)$, then $\left(p^{\prime}, q^{\prime}\right)$ is compatible with $(p, q)$.

Let $G$ be $\boldsymbol{P}$-generic over $M$, and let $A$ be the generic Souslin subset of $X$ determined by $G$. We first show that $M[G] \vDash^{\prime \prime} A$ is not Borel in $X^{\prime \prime}$. Suppose on the contrary that $\exists \tau, w B(v, \omega) a \boldsymbol{\Sigma}_{\beta}^{0}$ predicate with parameters in $M$, and $\boldsymbol{r} \in \boldsymbol{P}$ such that

$$
r \Vdash " \forall x \in X(x \in A \text { iff } B(\tau, x)) " .
$$

By c.c.c. we can find $\alpha<\omega_{1}$ such that $|\tau|_{\alpha}=0,|r|_{\alpha}=0$, and $\beta<\alpha$. Let $\gamma$ be any countable ordinal greater than $\alpha+\omega$. Extend $r=$ $(p, q)$ by adding $p_{r}(\phi)=1$ to $p$, and call the result $r_{1}$. By this addition, $r_{1} \Vdash " x \in A^{\prime \prime}$, so $r_{1} \Vdash^{\prime \prime} B\left(\tau, x_{\gamma}\right)^{\prime \prime}$, so there exists $r_{2}$ compatible with $r_{1}$ such that $\left|r_{2}\right|_{\alpha}<\beta$ and $r_{2} \Vdash^{\prime \prime} B\left(\tau, x_{\gamma}\right) "$. But since $\gamma>\alpha+\omega$ and $\left|r_{2}\right|_{\alpha}<\beta<\alpha$, it follows that $\exists r_{3} \geqq r_{2}$ such that $p_{r}^{3}(\phi)=0$ and 
thus $\Vdash^{\prime \prime} x_{r} \notin A^{\prime \prime}$. This is a contradiction since $r_{3}$ and $r_{1}$ are compatible (since $r_{2}$ and $r_{1}$ are compatible).

Now let us prove something a little stronger. Let $M \vDash " H \subseteq$ $P(X),|H| \leqq \omega^{\prime \prime}$, then, we claim $M[G] \vDash " A \notin B(H)$ (the $\sigma$-algebra generated by $H)^{\prime \prime}$.

Work in $M$. Let $H=\left\{A_{n}: n<\omega\right\}$ and define $K: X \rightarrow 2^{\omega}$ by $K(x)(n)=1$ iff $x \in A_{n}$. Let $Y$ be the range of $K$, then $K$ has the property that it maps the $\sigma$-algebra generated by $H$ into the Borel subsets of $Y$.

For any $C \in B(H)^{M[G]} \exists B$ Borel subset of $Y$, and $p \in \boldsymbol{P}$ such that

$$
p \Vdash^{\prime \prime} \forall x \in X(x \in C \quad \text { iff } \quad K(x) \in B)^{\prime \prime} .
$$

The preceding proof now goes through. Finally we are ready to state the theorem.

THEOREM 3. It is consistent with ZFC that there does not exist $H \subseteq P\left(2^{\omega}\right)$ countable such that every analytic set is in the $\sigma$-algebra generated by $H$.

Proof. Let $M, X$, and $\boldsymbol{P}$ be as above. Working in $M$ let $\left\{\boldsymbol{P}_{\alpha}: \alpha<\omega_{2}^{M}\right\}$ be a set of isomorphic copies of $\boldsymbol{P}$. Force with $\Sigma\left\{\boldsymbol{P}_{\alpha}\right.$ : $\left.\alpha<\omega_{2}^{M}\right\}$. Let $\left\langle G_{\alpha} . \alpha<\omega_{2}^{M}\right\rangle$ be generic over $M$. If $M\left[G_{\alpha}: \alpha<\omega_{2}^{M}\right] \vDash$ " $H \subseteq P\left(2^{\omega}\right),|H| \leqq \omega^{\prime \prime}$ then by c.c.c. $\exists \alpha_{0}<\omega_{2}^{I I}$ such that $\{B \cap X$ : $B \in H\} \in M\left[G_{\alpha}: \alpha \neq \alpha_{0}\right]$. Let $M\left[G_{\alpha}: \alpha \neq \alpha_{0}\right]$ be the new ground model and $\hat{A}$ the analytic set created by $\boldsymbol{P}_{\alpha_{0}}$. Note that although $\boldsymbol{P}_{\alpha_{0}}$ is not the same as adding Cohen reals, because of its finite nature it is the same partial order whether defined in $M$ or any extension of $M$ (e.g., $M\left[G_{\alpha}: \alpha \neq \alpha_{0}\right]$ ). We have already noted that $\hat{A} \cap X$ is not in the $\sigma$-algebra generated by $\{B \cap X: B \in H\}$ and therefore $\hat{A}$ is not in the $\sigma$-algebra generated by $H$.

2. Making subsets generic Souslin sets. Let $\Sigma$ be the set of countable successor ordinals greater than two. As in $\S 1$ let $X^{*}=$ $\left\{x_{\alpha}: \alpha \in \Sigma\right\} \subseteq 2^{\omega}$ and $F: 2^{\omega} \rightarrow 2^{\left(\omega \omega^{<\omega)}\right.}$ be the map such that $\forall \alpha \in \Sigma, F\left(x_{\alpha}\right)$ is a normal $\alpha$-tree $T_{\alpha}$. For $i=0$ or 1 and $T \subseteq \omega^{<\omega}$ define:

$$
\boldsymbol{P}^{i}(T)=\{p \in \boldsymbol{P}(T): \exists \hat{p} \text { an extension of } p, \hat{p}(\phi)=i\} .
$$

It is easy to check that for any $G$ which is $\boldsymbol{P}^{i}(T)$-generic over $M$, $G(\phi)=i$. Given $Z \cong \Sigma$ define $\boldsymbol{P}(Z)$ a suborder of $\boldsymbol{P}$ by $(p, q) \in$ $\boldsymbol{P}(Z)$ iff $(p, q) \in \boldsymbol{P}$ and $\forall \alpha \in \Sigma$

(a) if $\alpha \in Z$ then $p_{\alpha} \in \boldsymbol{P}^{0}\left(T_{\alpha}\right)$;

(b) if $\alpha \notin Z$ then $p_{\alpha} \in P^{1}\left(T_{\alpha}\right)$.

As before for $G \boldsymbol{P}(Z)$-generic over $M$, in $M[G],\left\{x_{\alpha}: \alpha \in Z\right\}$ is 
analytic in $X^{*}$. The reason for $\Sigma$ will be evident in the proof of Lemma 5.

THEOREM 4. There exist a generic extension $N$ of $M$ such that $N \vDash$ "Every subset of $X^{*}$ is analytic in $X^{*}$ but some subset of $X^{*}$ is not Borel in $X^{* \prime \prime}$.

Proof. $N$ will be obtained by iterating with finite support $\boldsymbol{P}(Z)$. Since each $\boldsymbol{P}(Z)$ is a relatively simple suborder of $\boldsymbol{P}$ we can give the following simpler definition. We assume $M \vDash " 2^{\omega_{1}}=\omega_{2}^{\prime \prime}$. Let $\boldsymbol{Q}=\sum_{\alpha<\omega_{2}} \boldsymbol{P}_{\alpha}$ as in $\S 1$ and for $p \in \boldsymbol{Q}$ define $\operatorname{supp}(p)=\left\{\alpha<\omega_{2}: p(\alpha) \neq 0\right\}$. Let $A_{\alpha}$ for $\alpha<\omega_{2}$ list with $\omega_{2}$ repetitions all maps $A: \omega_{1} \rightarrow[Q]^{\leqq \omega}$. Inductively define $\boldsymbol{Q}_{\alpha} \leqq \boldsymbol{Q}$ for $\alpha<\omega_{2}$. For $\alpha=0$ let $\boldsymbol{Q}_{\alpha}=\{p \in \boldsymbol{Q}$ : $\operatorname{supp}(p)=\{0\}\}$ (i.e., $\boldsymbol{Q}_{0}=\boldsymbol{P}$ ). For all $\alpha \boldsymbol{Q}_{\alpha} \leqq\{p \in \boldsymbol{Q}: \operatorname{supp}(p) \subseteq \alpha\}$. For $\alpha$ a limit ordinal let $\boldsymbol{Q}_{\alpha}=\cup\left\{\boldsymbol{Q}_{\beta}: \beta<\alpha\right\}$. For $\alpha+1$ let $G_{\alpha}$ be $\boldsymbol{Q}_{\alpha^{-}}$ generic over $M$ and let $Z_{\alpha}=\left\{\beta \in \Sigma: A_{\alpha}(\beta) \cap G_{\alpha} \neq \phi\right\}$. Then

$$
\begin{aligned}
\boldsymbol{Q}_{\alpha+1}= & \left\{p \in \boldsymbol{Q} \mid p\left\lceil\alpha \in \boldsymbol{Q}_{\alpha}, p\left\lceil\alpha \Vdash \boldsymbol{Q}_{\alpha} " p(\alpha) \in \boldsymbol{P}\left(Z_{\alpha}\right)^{\prime \prime},\right.\right.\right. \\
& \text { and } \operatorname{supp}(p) \leqq \alpha+1\} .
\end{aligned}
$$

(Of course by $p \nmid \alpha$ here we mean that condition in $\boldsymbol{Q}$ whose restriction to $\alpha$ is the same as $p$ 's and whose support is contained in $\alpha$.)

Thus if $G_{\omega_{2}}$ is $\boldsymbol{Q}_{\omega_{2}}$ generic over $M$ then $M\left[G_{\omega_{2}}\right] \models$ "Every subset of $X^{*}$ is analytic in $X^{* \prime \prime}$. Work in $M$. Given $\alpha<\omega_{1}$ recall the definition $|p|_{\alpha}$ for $p \in \boldsymbol{P}$ given in $\S 1$. Given $K \subseteq \omega_{2}$ and $\alpha<\omega_{1}$ define a map $F: \boldsymbol{Q}_{\omega_{2}} \rightarrow \alpha \cup\{\infty\} \quad$ by $F(p)=\max \left\{|p(\delta)|_{\alpha}: \delta \in K\right\} \quad$ if $\operatorname{supp}(p) \subseteq K$ and the $\max$ is less than $\alpha$, and otherwise let $F(p)=\infty$. Denote $F(p)$ by $|p|(K, \alpha)$. For suitably chosen $K$ and $\alpha$ we will show $|p|(K, \alpha)$ is a rank function. Given $\Gamma \leqq \boldsymbol{Q}_{\omega_{2}}$ and $\theta$ a sentence we say $\Gamma$ decides $\theta$ iff $\forall p \in \boldsymbol{Q}_{\omega_{2}} \exists q \in \Gamma \quad p$ and $q$ are compatible, and $q \Vdash$ " $\theta^{\prime \prime}$ or $q \Vdash "-\theta^{\prime \prime}$.

Lemma 5. Suppose that $\forall \delta \in K \forall \beta<\alpha\left\{p \in \boldsymbol{Q}_{\delta}:|p|(K, \alpha)=0\right\}$ de ides " $\beta \in Z_{\dot{o}}^{\prime \prime}$. Then $|p|(K, \alpha)$ is a rank function.

Proof. We must show that given $p \in \boldsymbol{Q}_{\omega_{2}}$ and $1 \leqq \beta \leqq \alpha$ there exists $\hat{p} \in \boldsymbol{Q}_{\omega_{2}}$ compatible with $p,|\hat{p}|(K, \alpha) \leqq \beta$, and $\forall q \in \boldsymbol{Q}_{\omega_{2}}$ if $|q|(K, \alpha)<\beta$ and $\hat{p}$ and $q$ are compatible, then $p$ and $q$ are compatible.

Recall that in the proof that ||$_{\alpha}$ is a rank function on $\boldsymbol{P}$ we obtained for each $p \in \boldsymbol{P}$ a $\hat{p} \in \boldsymbol{P}$ such that:

(a) $|\hat{p}|_{\alpha} \leqq \beta$

(b) $\hat{p}$ and $p$ are compatible;

(c) $\forall q \in \boldsymbol{P}$ if $|q|_{\alpha}<\beta$ and $q$ and $\hat{p}$ are compatible, then $q$ and $p$ are compatible; 
(d) $\forall \gamma<\alpha, \hat{p}(\gamma)=p(\gamma)$.

Given $p \in \boldsymbol{Q}_{\omega_{2}}$ define $\hat{p}$ by letting $\forall \delta \notin K, \hat{p}(\delta)=0$ and $\forall \delta \in K, \hat{p}(\delta)$ is the condition in $\boldsymbol{P}$ obtained above for $p(\delta)$. We show that $\hat{p} \in \boldsymbol{Q}_{\omega_{2}}$. Suppose not and let $\delta$ be the least such that $\hat{p} \uparrow \delta$ does not force " $\hat{p}(\delta) \in \boldsymbol{P}\left(Z_{\hat{o}}\right)$ ". Clearly $\delta \in K$. Let $\hat{p}(\delta)=\left(p^{\prime}, q\right)$. Then there must be some $\gamma \in \Sigma$ such that $p_{\gamma}^{\prime} \notin \boldsymbol{P}^{0}\left(T_{\gamma}\right)$ or $p_{\gamma}^{\prime} \notin \boldsymbol{P}^{1}\left(T_{\gamma}\right)$, and $\hat{p} \uparrow \delta$ does not force " $\gamma \notin Z_{\dot{o}}^{\prime \prime}$ respectively " $\gamma \in Z_{j}^{\prime \prime}$. If $p_{\gamma}^{\prime} \notin \boldsymbol{P}^{0}\left(T_{\gamma}\right)$ then $\phi \in \operatorname{dom}\left(p_{\gamma}^{\prime}\right)$ and $p_{\gamma}^{\prime}(\phi)=1$. If $p_{\gamma}^{\prime} \notin \boldsymbol{P}^{1}\left(T_{\gamma}\right)$ then either $\phi \in \operatorname{dom}\left(p_{\gamma}^{\prime}\right)$ and $p_{\gamma}^{\prime}(\phi)=0$ or $\exists n<$ $\omega,\langle n\rangle \in \operatorname{dom}\left(p_{\gamma}^{\prime}\right)$ and $p_{\gamma}^{\prime}(\langle n\rangle)=1$. Since $\gamma \in \Sigma$ it is a successor ordinal. Since $|p(\delta)|_{\alpha} \leqq \beta<\alpha$ and $|\langle n\rangle|_{T} \geqq \gamma-1$ it must be that $\gamma<\alpha$. By the properties of $K$ and $\alpha, \exists q \in \dot{Q}_{\hat{o}},|q|(K, \alpha)=0, q \Vdash^{\prime \prime} \gamma \notin Z_{\hat{o}}^{\prime \prime}$ (respectively " $\left.\gamma \in Z_{o}^{\prime \prime}\right)$, and $q$ is compatible with $\hat{p} \uparrow \delta$. But since $q$ is compatible with $\hat{p} \uparrow \delta$, it is compatible with $p \uparrow \delta$. This is a contradiction, since by $(\mathrm{d}) q \Vdash^{\prime \prime} \hat{p}(\delta) \notin \boldsymbol{P}\left(Z_{\hat{o}}\right)$ ".

If $A$ is the analytic subset of $X^{*}$ which is created at the first step, then $A$ is not Borel in $X^{*}$ in the model $M\left[G_{\omega_{2}}\right]$. To see this suppose not and $\exists p \in \boldsymbol{Q}_{\omega_{2}}$

$$
p \mathbb{H}^{\prime \prime} \forall x \in X^{*}\left(x \in A \text { iff } x \in B_{\tau}\right)^{\prime \prime}
$$

where $B_{\tau}$ is a $\boldsymbol{\Sigma}_{\beta}^{j}$ set with parameter $\tau \in 2^{\prime}$. Using the c.c.c. of $\boldsymbol{Q}_{\omega_{2}}$ it is easy to obtain $K \subseteq \omega_{2}$ countable, $0 \in K$, and $\alpha<\omega_{1}$ with $\beta<\alpha$, such that $|p|(K, \alpha)=0,|\tau|(K, \alpha)=0$, and $K$ and $\alpha$ satisfy the requirements set down in Lemma 5 . As in $\S 1$ this leads to a contradiction.

3. Abstract Souslin sets. Recall that $R=\left\{A \times B: A, B \cong 2^{\omega}\right\}$, $B(R)$ is the $\sigma$-algebra generated by $R$, and $S(R)$ the family of sets which are gotten by applying the Souslin operation to sets in $B(R)$.

THEOREM 6. It is consistent with ZFC that $S(R)=P\left(2^{\omega} \times 2^{\omega}\right) \neq$ $B(R)$.

The model used will be a minor modification of the one obtained in $\S 2$.

Lemma 7. Suppose $X \subseteq 2^{\omega},|X|=\left|2^{\omega}\right|$, and every subset of $X$ of cardinality less than $\left|2^{\omega}\right|$ is analytic in $X$. Then $S(R)=P\left(2^{\omega} \times 2^{\omega}\right)$.

Proof. Let $\kappa=\left|2^{\omega}\right|$ and $X=\left\{x_{\alpha}: \alpha<\kappa\right\}$. Since $S(R)$ is closed under finite union, it is enough to show that any $Y \cong \kappa^{2}$ with the property that $\langle\alpha, \beta\rangle \in Y \rightarrow \alpha \leqq \beta$, is in $S(R)$. For each $\beta$ let $X_{\beta}=$ $\left\{x_{\alpha}:\langle\alpha, \beta\rangle \in Y\right\}$. For each $\beta$ and $s \in \omega^{<\omega}$ let $C_{s}^{\beta}$ be a closed subset of $X$ such that $X_{\beta}=\bigcup_{f \in \omega^{\omega}} \bigcap_{n<\omega} C_{f \nmid n}^{\beta}$. 
For each $s \in \omega^{<\omega}$ define $B_{s}=\left\{\langle\alpha, \beta\rangle: x_{\alpha} \in C_{s}^{\beta}\right\}$. Since $Y=$ $\bigcup_{f \in \omega^{\omega}} \bigcap_{n<\omega} B_{f \mid n}$ it is enough to check that each $B_{s} \in B(R)$. Fix $s \in$ $\omega^{<\omega}$ and let $\left\{D_{n}: n<\omega\right\}$ be an open basis for $X$. For each $\beta$ define $y_{\beta}(n)=1$ iff $D_{n} \cap C_{s}^{\beta}=\phi . \quad$ It follows that $\alpha \in C_{s}^{\beta}$ iff $\forall n$ (if $y_{\beta}(n)=1$ then $\left.\alpha \notin D_{n}\right)$. Letting $E_{n}=\left(D_{n} \times X\right) \cup\left(D_{n} \times\left\{\beta: y_{\beta}(n)=0\right\}\right)$ we have that $B_{s}=\bigcap_{n<\omega} E_{n}$.

Lemma 8. Suppose $F: X \rightarrow Y$ is $1-1$ and $\forall U$ open in $Y F^{-1}(U)$ is Borel in $X$. If every subset of $Y$ is analytic in $Y$ then every subset of $X$ is analytic in $X$.

Proof. Given $A \leqq X$ let $B=F^{\prime \prime} A$. Then there are Borel subsets of $Y, B_{s}$ for $s \in \omega^{<\omega}$ such that $B=\bigcup_{f \in \omega^{\omega}} \bigcap_{n<\omega} B_{f i n}$. Let $A_{s}=F^{-1}\left(B_{s}\right)$, then $A_{s}$ is Borel in $X$ and $A=\bigcup_{f \in \omega^{\omega}} \bigcap_{n<\omega} A_{f \backslash n}$.

We now prove Theorem 2. Let $M$, the ground model of ZFC in $\S 2$, be a model of $M A+2^{w}=\omega_{2}$. We first show that for $G_{\omega_{2}} \boldsymbol{Q}_{\omega_{2}}$ generic over $M, M\left[G_{\omega_{2}}\right]$ models that $S(R)=P\left(2^{\omega} \times 2^{r}\right)$. Working in $M$ for any $Z, W \cong 2^{\omega}$ with $|Z|=|W|=\omega_{1}$, if $F: Z \rightarrow W$ is any 1- 1 map then by Silver's lemma (see [6]) for every $U$ open in $W, F^{-1}(U)$ is Borel in $Z . \quad F$ still has this property in any extension of $M$ since $W$ is second countable and $M$ contains an open basis for $W$. Working in $M$ there exists $X \cong 2^{\omega}$ such that $|X|=\omega_{2}$ and $\forall Y \subseteq X$ if $|Y| \leqq \omega_{1}$ then $Y$ is Borel in $X$ (a generalized Luzin set is such an example, see [9]). We claim that in $M\left[G_{\omega_{2}}\right]$ every subset of $X$ of size $\leqq \omega_{1}$ is analytic in $X$ and thus by Lemma $7, S(R)=$ $P\left(2^{\omega} \times 2^{\omega}\right)$. Working in $M\left[G_{\omega_{2}}\right]$ for any $Z \cong X$ if $|Z| \leqq \omega_{1}$ then $\exists Y \in M Z \subseteq Y$ and $|Y| \leqq \omega_{1}$. Letting $F: Y \rightarrow X^{*}$ be any $1-1$ map in $M$ we have by Lemma 8 that every subset of $Y$ is analytic in $Y$, and since $Y$ is Borel in $X, Z$ is analytic in $X$.

We next want to show that in $M\left[G_{\omega_{2}}\right], P\left(2^{\omega} \times 2^{\omega}\right) \neq B(R)$. It is enough to show that in $M\left[G_{\omega_{2}}\right]$ there does not exist a countable $H \cong P\left(X^{*}\right)$ such that $B(H)=P\left(X^{*}\right)$. To see that this suffices let $\left\{X_{\alpha}: \alpha<\omega_{2}\right\}=P\left(X^{*}\right)$ and let $Y=\left\{(x, \alpha): x \in X_{\alpha}\right\} \cong X^{*} \times \omega_{2}$. If $Y$ is in the $\sigma$-algebra generated by $\left\{A_{n} \times B_{n}: n<\omega\right\}$ then $B\left(\left\{A_{n}: n<\omega\right\}\right)=$ $P\left(X^{*}\right)$. Just show by induction that $\forall K \in B\left(\left\{A_{n} \times B_{n}: n<\omega\right\}\right) \forall \beta<\omega_{2}$ $\left\{x \in X^{*}:(x, \beta) \in K\right\} \in B\left(\left\{A_{n}: n<\omega\right\}\right)$.

By the technique of $\S 1$ and $\S 2$ we note that in $M$ there is no countable $H \cong P\left(X^{*}\right)$ such that the generic Souslin set created at the first step is in $B(H)$. Note that for $Z=\phi$ and $G P(Z)$-generic over $M$ the set $A=\left\{x_{\alpha} \in X^{*}: G_{\alpha}(\langle 0\rangle)=1\right\}$ is also a generic Souslin set over $M$. This is because the requirement that $G_{\alpha}(\dot{\phi})=0$ puts no constraint on the value of $G_{\alpha}(\langle 0\rangle)$. 
4. Remarks. (1) In the model used for Theorem 1 one can show that there does not exist any $H \subseteq P\left(2^{\omega}\right),|H|<\left|2^{\omega}\right|$, such that every analytic subset of $2^{\omega}$ is in $B(H)$. Note also that $\omega_{2}$ can be replaced by any $\kappa>\omega_{1}$ of uncountable cofinality. Also in this model it is true that the universal $\Sigma_{1}^{1}$ subset of $2^{\omega} \times 2^{\omega}$ is not in the $\sigma$ algebra generated by the abstact rectangles.

(2) It is not hard to modify the technique of $\S 2$ to get it consistent with ZFC that $\exists X \cong 2^{\omega}|X|=\omega_{2}$ (or even $|X|=\boldsymbol{\aleph}_{\omega_{1}}$ ) such that every subset of $X$ is analytic in $X$ but not every subset of $X$ is Borel in $X$.

(3) $X^{*}$ in $\S 2$ has Baire order $\omega_{1}$ in $M\left[G_{\omega_{2}}\right]$.

(4) In [5] Kunen showed that if one adds $\omega_{2}$ Cohen reals to a model of $\mathrm{CH}$ then $\left\{(\alpha, \beta): \alpha<\beta<\omega_{2}\right\}$ is not in the $\sigma$-algebra generated by $\left\{A \times B: A \subseteq \omega_{2}, B \subseteq \omega_{2}\right\}$. In the same model (actually $\mathrm{CH}$ is not necessary in ground model) there is a subset of $\omega_{1} \times \omega_{2}$ not in the $\sigma$-algebra generated by $\left\{A \times B: A \subseteq \omega_{1}, B \cong \omega_{2}\right\}$. To prove this it is enough to find $F \subseteq P\left(\omega_{1}\right)|F|=\omega_{2}$ such that there does not exist $H \subseteq P\left(\omega_{1}\right)$ countable with $F \cong B(H)$. Let $\boldsymbol{P}=\{p \mid p: F \rightarrow 2$, for some $\left.F \in\left[\omega_{1}\right]^{<\omega}\right\}$ and suppose $G$ is $\boldsymbol{P}$-generic over $M$. Let

$$
X=\left\{\alpha<\omega_{1} \mid G(\alpha)=1\right\}
$$

and note that for any $H \subseteq P\left(\omega_{1}\right)$ countable and in $M, M[G] \Vdash " X \notin$ $B(H)^{\prime \prime}$. This is because for any $Y \in B(H) \quad \exists t \in 2^{\omega} Y \in M[t]$.

(5) In [12] Rothberger showed that $2^{\omega}=\omega_{2}+2^{\omega_{1}}=\boldsymbol{\aleph}_{\omega_{2}}$ implies that not every subset of $\omega_{1} \times \omega_{2}$ is in the $\sigma$-algebra generated by $\left\{A \times B: A \subseteq \omega_{1}, B \cong \omega_{2}\right\}$. To see this let $G_{\alpha}$ for $\alpha<\boldsymbol{\aleph}_{\omega_{2}}$ list all countable subsets of $P\left(\omega_{1}\right)$. Since $\left|B\left(G_{\alpha}\right)\right| \leqq 2^{\omega}=\omega_{2}$ we can pick $K_{\alpha} \in P\left(\omega_{1}\right)$ for $\alpha<\omega_{2}$ such that $K_{\alpha} \notin \bigcup_{\beta<\omega_{\alpha}} B\left(G_{\beta}\right)$. It follows as in (4) that $\left\{(\beta, \alpha): \beta \in K_{\alpha}\right\}$ is not in the $\sigma$-algebra generated by $\left\{A \times B: A \subseteq \omega_{1}, B \cong \omega_{2}\right\}$.

\section{REFERENCES}

1. R. H. Bing, W. W. Bledsoe and R. D. Mauldin, Sets generated by rectangles, Pacific J. Math., 51 (1974), 27-36.

2. W. Fleissner and A. W. Miller, On Q-sets, Proc. Amer. Math. Soc., 78 (1980), 280-284.

3. R. W. Hansell, Some consequences of $(V=L)$ in the theory of analytic sets, to Proc. Amer. Math. Soc., 80 (1980), 311-319.

4. - letter to the author, July 1979.

5. K. Kunen, Inaccessibility properties of cardinals, Doctoral Dissertation, Stanford University (1968).

6. D. A. Martin and R. M. Solovay, Internal Cohen extensions, Ann. Math. Logic, 2 (1970), 143-178.

7. R. D. Mauldin, letter to author, March 1979.

8. - On rectangles and countably generated families, Fund. Math., 95 (1977), $129-139$. 
9. A. W. Miller, On the length of Borel hierachies, Ann. Math. Logic, 16 (1979), 233-267.

10. - On generating the category algebra and the Baire order problem, Bull. Acad. Polonaise, 27 (1979), 751-755.

11. F. Rothberger, A remark on the existence of a denumerable base for a family of functions, Canad. J. Math., 4 (1952), 117-119.

12. - On families of real functions with a denumerable base, Annals of Math., 45 (1944), 397-406.

13. S. Ulam, Problem 74, Fund. Math., 30 (1938), 365.

Received February 18, 1980 and in revised form July 28, 1980. Research partially supported by an NSF grant.

University of TeXas

Austin, TX 78712 



\section{PACIFIC JOURNAL OF MATHEMATICS}

\section{EDITORS}

DONALD BABBITT (Managing Editor)

University of California

Los Angeles, California 90024

Hugo RossI

University of Utah

Salt Lake City, UT 84112

C. C. MOORE and ANDREW OGG

University of California

Berkeley, CA 94720

\section{J. DugundJI}

Department of Mathematics University of Southern California Los Angeles, California 90007

R. Finn and J. Milgram Stanford University

Stanford, California 94305

\section{ASSOCIATE EDITORS}
R. ARENS
E. F. BeCKENBACH
B. H. Neumann
F. WoLF
K. YoSHIDA

\section{SUPPORTING INSTITUTIONS}

UNIVERSITY OF ARIZONA

UNIVERSITY OF BRITISH COLUMBIA

CALIFORNIA INSTITUTE OF TECHNOLOGY

UNIVERSITY OF CALIFORNIA

MONTANA STATE UNIVERSITY

UNIVERSITY OF NEVADA, RENO

NEW MEXICO STATE UNIVERSITY

OREGON STATE UNIVERSITY
UNIVERSITY OF OREGON

UNIVERSITY OF SOUTHERN CALIFORNIA

STANFORD UNIVERSITY

UNIVERSITY OF HAWAII

UNIVERSITY OF TOKYO

UNIVERSITY OF UTAH

WASHINGTON STATE UNIVERSITY

UNIVERSITY OF WASHINGTON 


\section{Pacific Journal of Mathematics}

\section{Vol. 97, No. $1 \quad$ January, 1981}

Charles A. Asmuth and Joe Repka, Tensor products for $S L_{2}(\mathfrak{k})$. II.

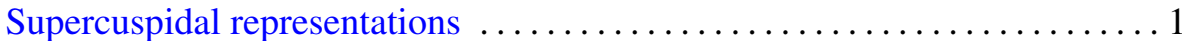

Joseph Barback, On finite sums of regressive isols . ................. 19

Matthew G. Brin and Daniel Russell McMillan, Jr., Generalized

three-manifolds with zero-dimensional nonmanifold set ............29

Kun Soo Chang, Converse measurability theorems for Yeh-Wiener space . . . 59

Christopher Brian Croke, A "maximal torus" type theorem for complete

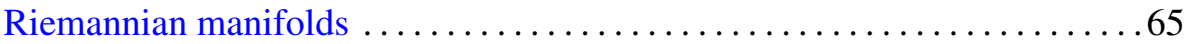

Gustave Adam Efroymson, Sums of squares in planar Nash rings . . . . . . 75

John Robert Fisher, Axiomatic radical and semisimple classes of rings . . . .81

Betty Kvarda, Consecutive integers for which $n^{2}+1$ is composite .......93

Roosevelt Gentry, New diagram proofs of the Hausdorff-Young theorem

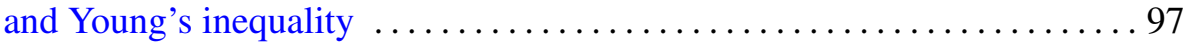

Patrick M. Gilmer, Topological proof of the $G$-signature theorem for $G$

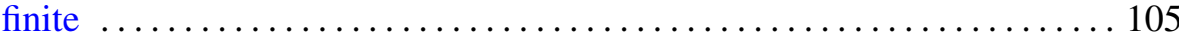

Chung Wei Ha, A noncompact minimax theorem .................. 115

James J. Hebda, Manifolds admitting taut hyperspheres ................ 119

Takayuki Kawada, Sample functions of Pólya processes ............. 125

Peter K. F. Kuhfittig, Common fixed points of nonexpansive mappings by

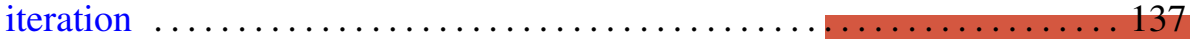

James Thomas Loats and Judith Roitman, Almost rigid Hopfian and dual

Hopfian atomic Boolean algebras .......................... 141

Roger McCann, On embedding semiflows into a radial flow on $l_{2} \ldots \ldots \ldots 151$

John McDonald, Closed orbits of convex sets of operators on the disk

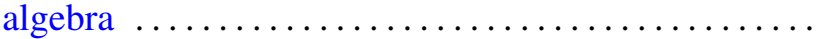

Mark D. Meyerson, Convexity and the table theorem .............. 167

Arnold William Miller, Generic Souslin sets . . . . . . . . . . . . . . . . 171

Takemi Mizokami, On the closed images of paracomplexes $\ldots \ldots \ldots \ldots \ldots 3$

Jagannadham Venkata Pakala and Thomas Stephen Shores, On

compactly packed rings $\ldots \ldots \ldots \ldots \ldots \ldots \ldots \ldots \ldots$

Andrew Pletch, Strong completeness in profinite groups

Wilbur Carrington Whitten, Inverting double knots

James Juei-Chin Yeh, Existence of strong solutions for stochastic

differential equations in the plane 